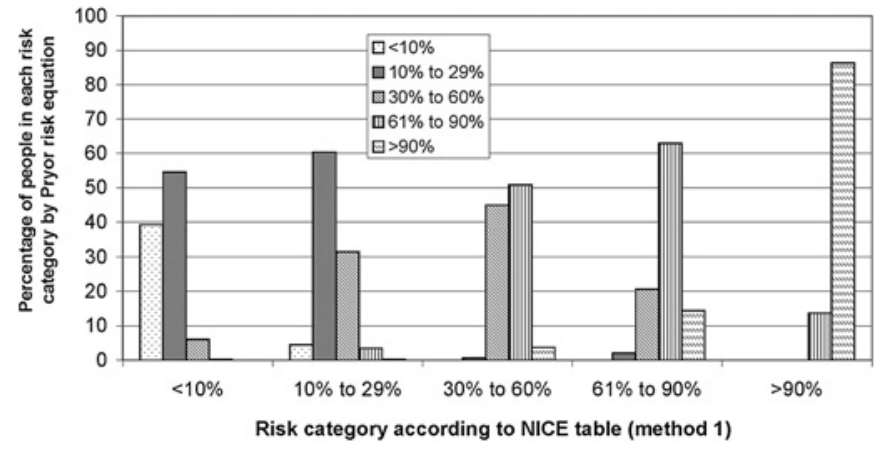

Abstract 140 Figure 1 Percentage of people in each risk category using the Pryor risk equation when initally categorised by the NICE table.

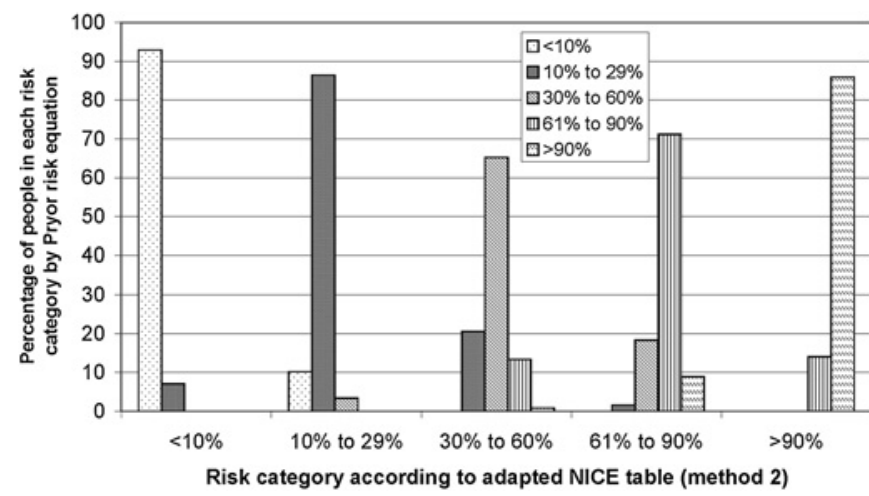

Abstract 140 Figure 2 Percentage of people in each risk category using the Pryor risk equation when initially categorised by the adapted NICE table.

Conclusion Use of the NICE table uncritically for assessing PTL of $\mathrm{CAD}$ misclassifies a significant proportion of people for further investigation. It is important to include ECG data, interpolate risk factor information, and take into account actual age when using the table rather than the Pryor equation, use of which takes these factors into account automatically.

\section{CARDIAC TROPONIN REFLECTS SILENT MYOCARDIAL ISCHAEMIA IN PATIENTS WITH STABLE CORONARY ARTERY DISEASE}

doi:10.1136/heartjnl-2012-301877b.141

${ }^{1} \mathrm{~A} S$ V Shah, ${ }^{*}{ }^{1} \mathrm{~J}$ P Langrish, ${ }^{2} \mathrm{X} \mathrm{Li,}{ }^{2} \mathrm{~L}$ Jiang, ${ }^{1} \mathrm{D}$ E Newby, ${ }^{1} \mathrm{~N}$ L Mills. ${ }^{1}$ Royal Infirmary of Edinburgh, Edinburgh, UK; ${ }^{2}$ Fuwai Hospital, Beijing, China

Background Cardiac troponin is an independent predictor of cardiovascular mortality in patients with stable coronary artery disease, yet the factors that determine circulating troponin concentration in these patients are not known. We hypothesise that circulating cardiac troponin in patients with stable coronary heart disease is due to recurrent silent myocardial ischaemia.

Methods 98 patients with stable coronary artery disease $(62 \pm 7$ years) were assessed on two occasions at least 2 weeks apart. Ambulatory blood pressure and 12-lead Holter monitoring were recorded for 24-h and symptom severity was assessed using the Seattle Angina Questionnaire. Significant silent myocardial ischaemia was defined as horizontal or down sloping ST-segment depression of at least $1 \mathrm{~mm}$ lasting at least $60 \mathrm{~s}$. Cardiac troponin I was measured in serum using the ultra-sensitive Singulex Erenna System (Singulex Inc., Berkeley, California, USA) following each visit.

Results Cardiac troponin I concentrations were quantifiable above the limit of detection (LOD, $0.4 \mathrm{pg} / \mathrm{ml}$ ) in all patients with a mean concentration of $6.1 \mathrm{pg} / \mathrm{ml}(95 \%$ CI 4.6 to $7.6 \mathrm{pg} / \mathrm{ml})$. The interassay coefficient of variation (CV) was $11 \%$ at the LOD and was $17 \%$ between visits. Concentrations were above the 99th percentile of a healthy reference population $(10.1 \mathrm{pg} / \mathrm{ml})$ in $15 \%$ of patients. Few patients $(n=2)$ reported angina during either visit, but troponin concentrations were significantly higher in patients with silent myocardial ischaemia $(n=17)$ compared to those without $(16.1 \pm 23.0$ vs $5.1 \pm 7.9 \mathrm{pg} / \mathrm{ml} ; \mathrm{p}<0.0001)$. Troponin concentrations were associated with both maximum ST-segment depression $(\mathrm{r}=-0.15, \mathrm{p}=0.03)$ and total ischaemic burden over the $24-\mathrm{h}$ period $(\mathrm{r}=-0.19, \mathrm{p}=0.009)$.

Conclusion Cardiac troponin concentrations above the recommended threshold for the diagnosis of myocardial infarction are present in as many as one in six patients with stable coronary artery disease and reflect in part reversible silent myocardial ischaemia. These findings have major implications for the diagnosis of myocardial infarction.

\section{ONSET OF PREECLAMPSIA IS PRECEDED BY STRUCTURAL CAPILLARY RAREFACTION}

doi:10.1136/heartjnl-2012-301877b.142

${ }^{1} \mathrm{~V}$ Nama, ${ }^{2} \mathrm{~T}$ Manyonda, ${ }^{3} \mathrm{~J}$ Onwude, ${ }^{1} \mathrm{~T}$ F T Antonios. ${ }^{*}$ Blood Pressure Unit 8 Department of Clinical Sciences, St. George's, University of London, London, UK; ${ }^{2}$ Department of Obstetrics and Gynaecology, St. George's Hospital NHS Trust, London, UK; ${ }^{3}$ Department of Obstetrics and Gynaecology, Springfield Hospital, Lawn Lane, Chelmsford, UK

Introduction Microvascular rarefaction, defined as reduced vascular density, is a consistent finding in hypertension. Functional and structural capillary rarefaction occurs in individuals with sustained and borderline essential hypertension, and in their normotensive offspring. Women who develop preeclampsia are at increased risk of hypertension in later life. We hypothesised that capillary rarefaction precedes the onset of preeclampsia and could play a role in its pathogenesis.

Methods In this longitudinal cohort study we recruited 322 Caucasian women, of which 305 subjects completed the study. We used intravital video-microscopy to measure basal (ie, functional) and maximal (ie, structural) skin capillary densities according to a well-validated protocol and measured plasma angiogenic and anti-angiogenic factors. Subjects were studied at five consecutive visits.

Results Preeclampsia occurred in 16 women (mean onset at $35.6 \pm 4.8$ weeks) and 272 women had normal pregnancy. In women with normal pregnancy significant structural reduction in capillary density occurred at 27-32 weeks, which had resolved by the puerperium (mean change: -2.2 capillaries/field, 95\% CI -3.6 to -0.7$)$. In contrast, in women who developed preeclampsia, more significant structural rarefaction was observed earlier at 20-24 weeks (mean change: -6.1 capillaries/field, 95\% CI -9.2 to -2.9 ), which persisted into the puerperium. We also found that the change in soluble Endoglin from 11-16 weeks to 27-32 weeks was significantly correlated with the change in structural capillary density.

Conclusions This is the first study to show that significant structural capillary rarefaction precedes the onset of preeclampsia. Capillary rarefaction could play a role in the pathogenesis of this disease. 